\title{
Pedicle screw removal along the descending aorta with endovascular intervention: a case report
}

\begin{abstract}
Iatrogenic vascular injuries after spinal surgery have been reported in many literatures but it was a rare complications. We report one case of a 26 -year - old male who was involved in road traffic accident with multiple injuries: T3-T4 fracture, left proximal humerus fracture and 8th to 9th rib fractures. Patient underwent for spinal fixation from T1 to T8 on 5thof September 2019. After one month, patient started to have a dry gangrene in the left big toe. CT angiogram showed the pedicle screw was crossed the level of the descending aorta. On 27 th of November 2019, patient was operated for screw removal under angiography.
\end{abstract}

Keywords: pedicles, endovascular, aorta, gangrene
Volume 12 Issue 2 - 2020

\author{
Mohammed H Al-Rumaih,' Fahad K Al-Jaber,' \\ Abdullah F Arab, ${ }^{3}$ Hamad H Al-Dakhil Allah' \\ 'Department of Orthopaedic Surgery, Prince Sultan Military \\ Medical City, Saudi Arabia \\ ${ }^{2}$ Department of Surgery, Division of Vascular \& Endovascular \\ Surgery, Prince Sultan Military Medical City, Saudi Arabia \\ ${ }^{3}$ Department of Spine Surgery, Prince Sultan Military Medical \\ City, Saudi Arabia
}

\begin{abstract}
Correspondence: Mohammed Al-Rumaih, Department of Orthopaedic Surgery, Prince Sultan Military Medical City, PO Box 7897, Riyadh II I59, Riyadh, Saudi Arabia,

Tel +9661 | 47777| 14, Email mhmalrumaih@psmmc.med.sa
\end{abstract}

Received: March 31, 2020 | Published: April 14, 2020

\section{Introduction}

Vascular complications to major vessels after spinal fixation surgery are very rare. Papadoulas et al. ${ }^{1}$ have been reported a low incidence of around $0.01 \%$ in a report on vascular injury pertinent to lumbar intervertebral disc surgery followed up for 36 years. Penetration of the pedicle screw to the major vessels such as descending aorta can be manifested from immediate hemorrhage to pseudoaneurysm formation. ${ }^{2,3}$ There have been several reports published describing such cases and the various approaches treatment. However, the presentation and complications can be varied. Additionally, as vascular traumatic treatment has evolved to less invasive therapies, multiple methods for repair exist. It has been reported that the outcome of a vessel injury generally varies depending on the time taken to diagnose the injury, type of vascular injury, and time taken to take the patient to an emergency intervention. ${ }^{4,5}$

\section{Case presentation}

A 26-year-old male, soldier, involved in road traffic accident on 27th of August 2019 in the southern region of Saudi Arabia. The patient was transferred to the military hospital in the south and they stabilized him there and then transferred to our hospital on 29th of August 2019. The patient had multiple injuries: T3-T4 spinal fracture (Figure 1), left proximal humerus fracture (Figure 2), $8^{\text {th }} \&$ $9^{\text {th }}$ rib fractures with multiple lacerations. He underwent for left open reduction and internal fixation of the proximal humerus by using Proximal Humerus Internal Locking System "PHILOS" plating on 03-Sep-2019 (Figure 3A \& 3B). Also, the patient underwent spinal fixation for the fracture from T1 to T8on 05-Sep-2019 (Figure 3C). One month postoperatively, he started to have left big toe gangrene and vascular surgery was consulted. They recommended doing CT angiogram of the aortic arch and echocardiogram. CT showed that left pedicular screw of T6 and T8 appear lateral to the pedicle and the tip of the screws abutting the medial wall of the descending thoracic aorta at the posterior mediastinum but there is no obvious surrounding active haematoma or aortic thrombosis (Figure 4A \& 4B). Pre- operative assessment and measurement were done and it was showed: Whole length supposed to be covered: $10 \mathrm{~cm}$, Proximal landing zone on the sagittal view: $2.67 \mathrm{~cm}$, Distal landing zone on the sagittal view: $1.83 \mathrm{~cm}$, Proximal axial: $2.14 \mathrm{~cm}$ x $2.11 \mathrm{~cm}$, and distal axial: $1.92 \mathrm{~cm} \mathrm{x}$ $1.78 \mathrm{~cm}$.

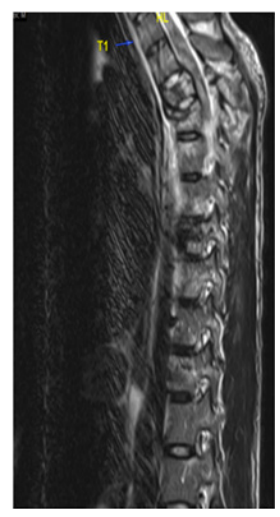

Figure I MRI sagittal cut of cervical and dorsal spine showed a compression fracture of 33 vertebral body with $50 \%$ reduction in its height and compression fracture of T4 involving only the superior endplate with retro-pulsed posterior vertebral margin of $\mathrm{T} 3$ causing cord contusion.

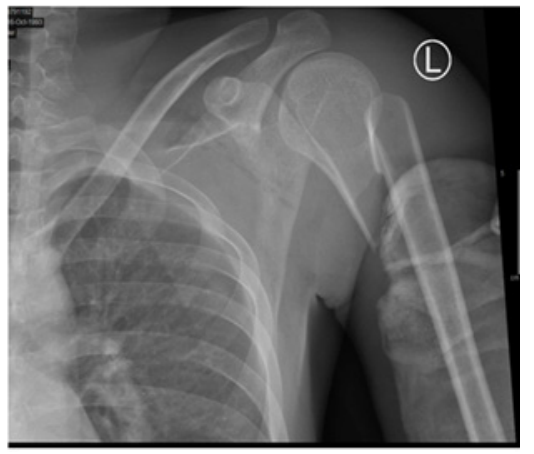

Figure 2 X-ray of left shoulder showed displaced proximal humerus fracture. 


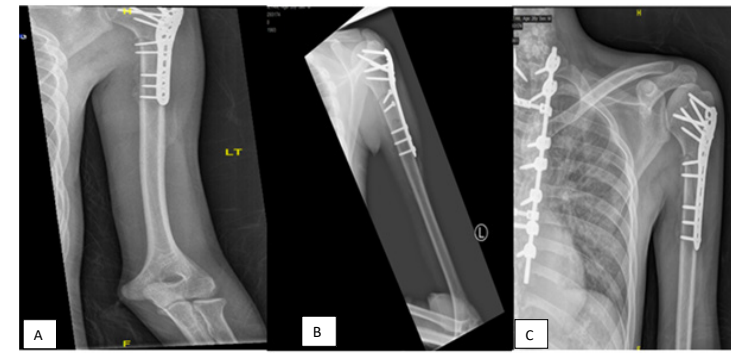

Figure 3 Anteroposterior (AP) \& lateral views of left humerus, post open reduction and internal fixation by PHILOS plate (05.Sep .2019) [A\&B ], with posterior spinal instrumentation from TI -T7 [C].
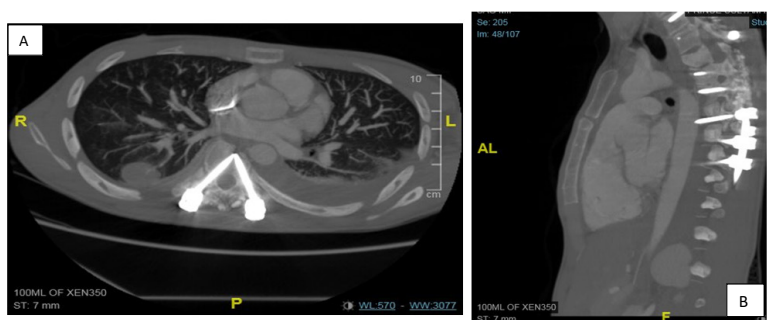

Figure 4 CT Axial cut (A) and Sagittal cut (B) showed The thoracic aorta appears intact however the left pedicular screw of T6 and T8 appear lateral to the pedicle and the tip of the screws abutting the medial wall of the descending thoracic aorta at the posterior mediastinum but there is no obvious surrounding active haematoma or aortic thrombosis. Whole length supposed to be covered: $10 \mathrm{~cm}$, Proximal landing zone on the sagittal view: $2.67 \mathrm{~cm}$, Distal landing zone on the sagittal view: $1.83 \mathrm{~cm}$, Proximal axial: $2.14 \mathrm{~cm}$ $\times 2.1 \mathrm{lcm}$, and distal axial: $1.92 \mathrm{~cm} \times 1.78 \mathrm{~cm}$.

On 27th of November 2019, patient underwent for removal of the pedicle screw under angiography which was a combined surgery between spine surgery and vascular surgery. Patient put in the lateral decubitus position and left pedicular screw of T6 and T8 were removed (Figure $5 \& 6$ ). There was no leakage with minimal blood loss. The items were used in this case:
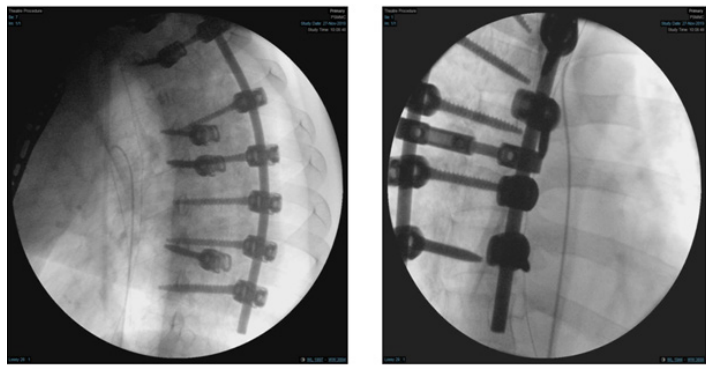

Figure $\mathbf{5}$ Intraoperative fluoroscopy during removal of the pedicle screw.
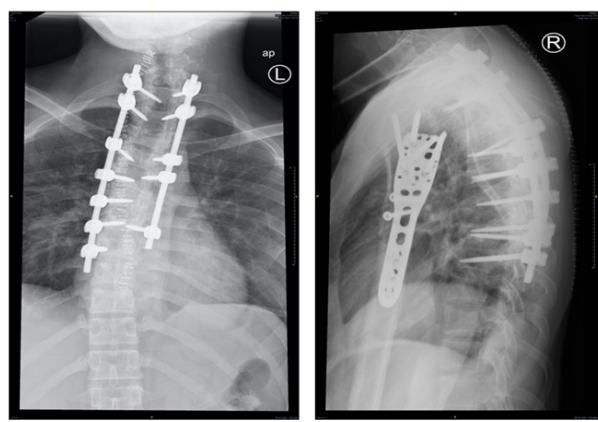

Figure 6 Postoperative $x$-ray of the spine after removal of left pedicular screw of $\mathrm{T} 6$ and $\mathrm{T} 8$. a. Two French sheaths / Size: 6 \&12

b. Multiple holes pigtail catheter

c. Terumo wire $0.035260 \mathrm{~cm}$

d. Super stiff wire $260 \mathrm{~cm}$

e. Aortic occlusion balloon ( coda balloon )

f. Thoracic aortic stent Gore $26 \mathrm{~mm} \times 10 \mathrm{~cm}$ ( standby )

g. Dry seal sheath 20 French

h. Two closure devices (proglide )

i. Power injector

j. Insufflator

k. Angiogram table

1. C-arm image intensifier

m. Angiographic puncture needle

\section{Discussion}

Most of literatures that describing of vascular complications after spinal surgery is limited to acute presentation. However there are some cases reported for delayed vascular complications after spinal instrumentation.

Been et al. ${ }^{2}$ reported one case of young patient known to have Marfan's syndrome \& scoliosis. Patient was operated at age of 20 years for scoliosis surgery and he underwent for spinal instrumentation through anterior approach from T12 to L3. At the age of 40 years, he came to clinic for follow-up and presented with exertional dyspnea. Saccular aneurysm of the descending aorta was detected during a routine screening and they did for him additional CT contract and they found the proximal screw of is directly against the aortic wall. The head of the screw had perforated the wall of the vessel and it produced a false aneurysm with a $5 \mathrm{~cm}$ diameter proximally. Patient underwent for endovascular intervention by using thoracic endovascular graft which was inserted through left common femoral artery to the descending aorta. The duration of the surgery was about 59 minutes with minimal blood loss and no leakage. Patient was discharged after 5 days of the surgery.

Matsuzaki et al. ${ }^{6}$ reported one case of an elderly patient who developed back pain and lower limb paresthesia for 2years. Patient was admitted for further managements and investigations. A plain roentgenogram as well as myelogram were done him. They showed 50 degree of kyphosis in T5 to T10 vertebrae and spondylosis and, it showed a compression at level of T8 on myelogram. So, the patient underwent for kyphosis surgery correction through anterior approach from T6 to T9. He came to clinic after 6 months for follow-up and they did for him CT scan and they found accidentally the screw penetrating the aorta at level of T6. Patient was readmitted again in the hospital for further investigations. The screw was removed through open thoracotomy and during intra-operatively they found the tip of screw was protruding from the aortic wall and the mural tissue of the vessel twisted around the wall. The aorta was resected about $8 \mathrm{~cm}$ and it was replaced by artificial blood vessel.

Minor et al. ${ }^{7}$ reported one case of an elderly patient who had history of chronic scoliosis and he underwent for surgical correction through posterior spinal instrumentation. Patient came to the clinic as a routine 
follow-up and they did for him CT scan and they found left pedicle screw of T5 was mispositioned and it had penetrated the thoracic aortic wall. After two days, patient underwent for open thoracotomy under direct fluoroscopic guidance and they inserted it through the left common femoral artery to the proximal part of descending aorta. The duration of the surgery was about 100 minutes with minimal blood loss and no leakage on the aortogram. Patient was discharged within 24 hours of the surgery without any complications.

\section{Conclusion}

Endovascular intervention for removal of the pedicle screw is a minimal invasive procedure and it decreased risk of morbidity and mortality postoperatively compared to open surgical approach. However, both of these surgical approaches have pros and cons depending on the patient's status, location of the injury, clinical presentation \& interval time between injury and repair. Early recognition is a cornerstone in the management to prevent vascular complications after spinal instrumentation.

\section{Acknowledgments}

None.

\section{Consent}

Written informed consent was obtained from the patient for publication of this case report and accompanying images. A copy of the written consent is available for review by the Editor-in-Chief of this journal on request. The authors certify that they have obtained all appropriate patient consent forms. The patient understands that his name and initials will not be published in the study.

\section{Conflicts of interest}

The authors declare there are no conflicts of interest.

\section{Funding}

The authors received no financial support for the research, authorship, and/or publication of this article.

\section{References}

1. Papadoulas S, Konstantinou D, Kourea H, et al. Vascular injury complicating lumbar disc surgery. A systematic review. Eur J Vasc Endovasc Surg. 2002;24(3):189-195.

2. Been HD, Kerkhoffs GM, Balm R. Endovascular graft for late iatrogenic vascular complication after anterior spinal instrumentation: a case report. Spine. 2006;31(22):E856-E858.

3. Higashino K, Katoh S, Sairyo K, et al. Pseudoaneurysm of the thoracoabdominal aorta caused by a severe migration of an anterior spinal device. Spine J. 2008;8(4):696-699.

4. Birkeland IW, Taylor T. Major vascular injuries in lumbar disc surgery. $J$ Bone Joint Surg Br. 1969;51(1):4-19.

5. Kim HS, Chong HS, Nanda A, et al. Vascular injury in thoracolumbar spinal surgeries and role of angiography in early diagnosis and management. J Spinal Disord Tech. 2010;23(6):418-424.

6. Matsuzaki H, Tokuhashi Y, Wakabayashi K, et al. Penetration of a screw into the thoracic aorta in anterior spinal instrumentation. A case report. Spine. 1993;18(15):2327-2331.

7. Minor ME, Morrissey NJ, Peress R, et al. Endovascular treatment of an iatrogenic thoracic aortic injury after spinal instrumentation: case report. J Vasc Surg. 2004;39(4):893-896. 